\title{
Controlling Unstable Discrete Chaos and Hyperchaos Systems
}

\author{
Xin Li, Suping Qian \\ School of Mathematics and Statistics, Changshu Institute of Technology, Changshu, China \\ Email: lovelixin0412@163.com
}

Received July 23, 2013; revised August 23, 2013; accepted August 30, 2013

Copyright (C) 2013 Xin Li, Suping Qian. This is an open access article distributed under the Creative Commons Attribution License, which permits unrestricted use, distribution, and reproduction in any medium, provided the original work is properly cited.

\begin{abstract}
A method is introduced to stabilize unstable discrete systems, which does not require any adjustable control parameters of the system. 2-dimension discrete Fold system and 3-dimension discrete hyperchaotic system are stabilized to fixed points respectively. Numerical simulations are then provided to show the effectiveness and feasibility of the proposed chaos and hyperchaos controlling scheme.
\end{abstract}

Keywords: 2-Dimension Discrete Fold System; 3-Dimension Discrete Hyperchaotic System; Lyapunov Stability Theory; Controlling Chaos

\section{Introduction}

In many engineering and other practical problems, chaos is undesirable and therefore needs to be controlled. Thus, a large number of control methods have been developed and are being applied to real systems [1-10]. The method given by Ott, Grebogi and Yorke (OGY) [1] is to stabilize an unstable orbit in the neighborhood of a hyperbolic fixed point by forcing the orbit onto the stable manifold. The method proposed by Romeiras, Grebogi, Ott and Dayawansa (RGOD) [2] is not yet suitable for controlling hyperchaos since the method changes the stability property of the fixed point completely. However, the method proposed by Yang, Liu and Jian-min Mao [11] gives a new idea to stabilize unstable orbits even if there is no preexisting stable manifold nearby. For a finitedimensional dynamical system, whose governing equations may or may not be analytically available, Yang, Liu and Mao show how to stabilize an unstable orbit in a neighborhood of a "fully" unstable fixed point. The advantage of this method is: only one of the unstable directions is to be stabilized via time-dependent adjustments of control parameters. The parameter adjustments can be optimized. Recently, Bu [12] and Li [13] stabilized unstable discrete systems by a method which does not require any adjustable control parameters of the system.

*The work was supported by the Special Funds of the National Natural Science Foundation of China (Grant No. 11141003).
Consider an $n$-dimensional dynamical system defined by

$$
x_{k+1}=F\left(x_{k}\right),
$$

where $x \in R^{n}$ is an $n$-dimensional vector, $F$ is a nonlinear vector valued function. Let $x_{f}$ be the fixed point of the map (1). To stabilize a chaotic orbit to this fixed point, we take a variable feedback control described by

$$
x_{k+1}=F\left(x_{k}\right)+M\left(F\left(x_{k}\right)-x_{k}\right),
$$

Define an infinitesimal deviation of $x_{k}$ from $x_{f}$ as $\delta x_{k}=x_{k}-x_{f}$. Then from Equation (2), one has

$$
\delta x_{k+1} \approx J \delta x_{k}+M(J-I) \delta x_{k},
$$

where $J=\left.\left(\partial F / \partial x_{k}\right)\right|_{x_{k}=x_{f}}$ is the Jacobian matrix of the original system $F$ evaluated at the fixed point $x_{f}$ and $I$ is the $n \times n$ identity matrix. The goal of controlling here is to make $\lim _{k \rightarrow \infty}\left|\delta x_{k}\right|=0$. For this aim, one requires

$$
\delta x_{k+1}=Q \delta x_{k},
$$

where $Q$ is an $n \times n$ matrix and takes the form

$$
Q=\left(\begin{array}{cc}
q_{1} & 0 \\
0 & q_{2}
\end{array}\right),
$$

where are constants. Substituting Equation (4) and Equation (5) into Equation (3) and eliminating $\delta x_{k}$, choosing one special form of the matrix $Q=q I, q \in(-1,1)$, one have 


$$
M=(q I-J)(J-I)^{-1}
$$

This needs to use numeric computation to do. Therefore the above scheme based on the symbolic numeric computation is summarized as follows.

Input:

1) The unstable system (1);

2) The system (2) with a variable feedback controller;

3) Choose the initial values of systems (2).

\section{Output:}

1) $J=\left.\left(\partial F / \partial x_{k}\right)\right|_{x_{k}=x_{f}}$;

2) $M$ in (6);

3) Deduce the system (2) according to the results (6);

4) Numerical simulations of the states $x_{k}$ when $k \rightarrow \infty$.

In this paper, we use the method to stabilize 2-dimension discrete Fold system [14] and 3-dimension discrete hyperchaotic system due to Wang [15] to fixed points respectively.

\section{Stabilizing 2-Dimensional Discrete Fold System}

Using the above method, we stabilize 2-dimension discrete Fold system presented as:

$$
\left\{\begin{array}{l}
x_{1}(k+1)=x_{2}(k)+\alpha x_{1}(k) \\
x_{2}(k+1)=\beta+x_{1}^{2}(k)
\end{array},\right.
$$

where $\alpha, \beta$ are the parameters, and we choose $\alpha=-0.1$, $\beta=-1.7$.

In the following based on the method mentioned above, we will make the Fold system stabilize at the fixed point. It is easy to get the two fixed points $(1.965097170$, $2.161606887)$, and $(-0.8650971698,-0.9516068868)$ of Equation (7). The Jacobian matrix corresponding the fixed point $\left(x_{1 f}, x_{2 f}\right)$ is

$$
J=\left(\begin{array}{cc}
\alpha & 1 \\
2 x_{1 f} & 0
\end{array}\right) .
$$

From (6) one can have

$$
M=\left(\begin{array}{cc}
\frac{q-\alpha-2 x_{1 f}}{\alpha-1-2 x_{1 f}} & \frac{-1+q}{\alpha-1-2 x_{1 f}} \\
\frac{2 x_{1 f}(q-1)}{\alpha-1-2 x_{1 f}} & -\frac{2 x_{1 f}+q \alpha-q}{\alpha-1-2 x_{1 f}}
\end{array}\right),
$$

here we choose

$\left(x_{1 f}, x_{2 f}\right)=(1.965097170,2.161606887)$ as our research object. Choosing the parameter $q=0.5$, and $q=0.3$ respectively, one gets

$$
\begin{aligned}
& M_{1}=\left(\begin{array}{cc}
-1.176666313 & -0.1766663133 \\
-0.6943329445 & -1.194332944
\end{array}\right), \\
& M_{2}=\left(\begin{array}{cc}
-1.247332838 & -0.2473328387 \\
-0.9720661223 & -1.272066122
\end{array}\right) .
\end{aligned}
$$

From (2), respectively substitute (10) into (7), we can obtain

$$
\left\{\begin{aligned}
x_{1}(k+1)= & x_{2}(k)+\alpha x_{1}(k)-1.76666313\left[x_{2}(k)+\alpha x_{1}(k)-x_{1}(k)\right] \\
& -0.1766663133\left[\beta+x_{1}^{2}(k)-x_{2}(k)\right] \\
x_{2}(k+1)= & \beta+x_{1}^{2}(k)-0.6943329445\left[x_{2}(k)+\alpha x_{1}(k)-x_{1}(k)\right] \\
& -1.194332944\left[\beta+x_{1}^{2}(k)-x_{2}(k)\right]
\end{aligned}\right.
$$

and

$$
\left\{\begin{aligned}
x_{1}(k+1)= & x_{2}(k)+\alpha x_{1}(k)-1.247332838\left[x_{2}(k)+\alpha x_{1}(k)-x_{1}(k)\right] \\
& -0.2473328387\left[\beta+x_{1}^{2}(k)-x_{2}(k)\right] \\
x_{2}(k+1)= & \beta+x_{1}^{2}(k) 0.9720661223\left[x_{2}(k)+\alpha x_{1}(k)-x_{1}(k)\right] \\
& -1.272066122\left[\beta+x_{1}^{2}(k)-x_{2}(k)\right]
\end{aligned}\right.
$$

In the following, we give the orbit of 2-dimension discrete Fold system before being stabilized in Figure 1(a). And in Figure 1(b), three orbits starting from different initial points are stabilized to the fixed point (1.965097170, 2.161606887). It is shown that the unstable orbit is stabilized to the desired fixed point monotonically. Then the orbits stabilized of $x_{k}$ and $y_{k}$ versus $t_{k}$ are depicted contrasting with the ones before being stabilized in Figures 2 and 3, respectively.

\section{Stabilizing 3-Dimension Discrete Hyperchaotic System}

In this section, we consider 3-dimension discrete hyperchaotic system 


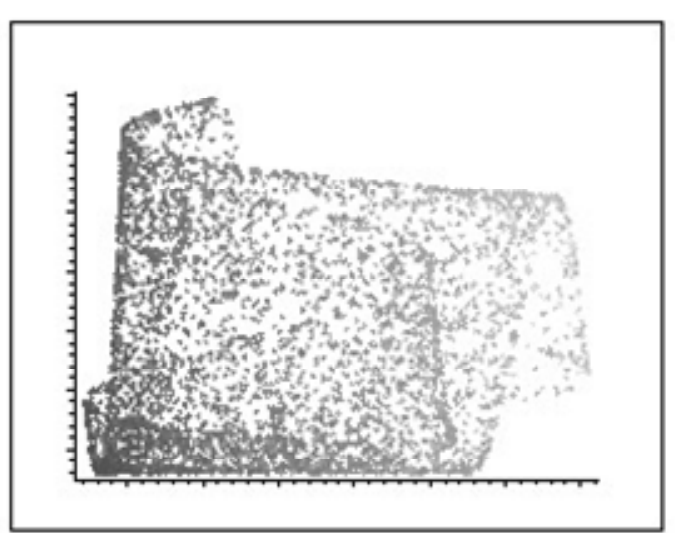

(a)

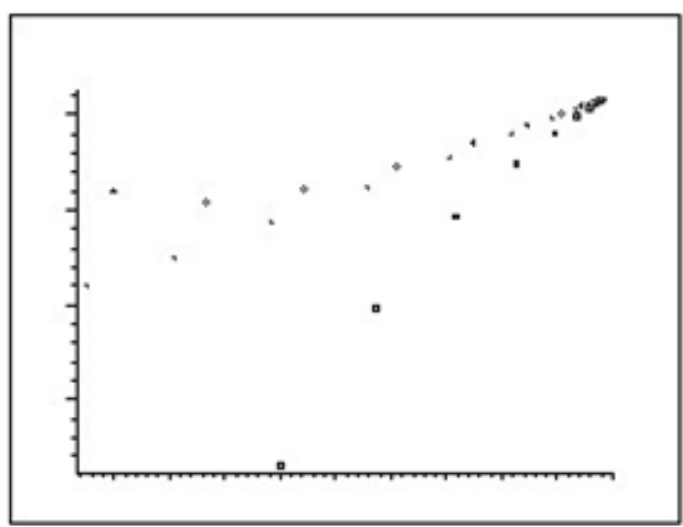

(b)

Figure 1. (a) 2-dimension discrete Fold system; (b) Three orbits starting from different initial points are stabilized to the fixed point (1.965097170, 2.161606887), for $q=0.5$.

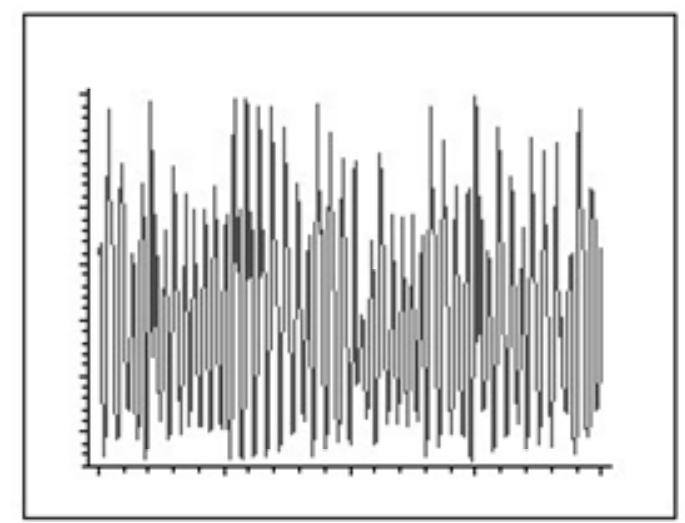

(a)

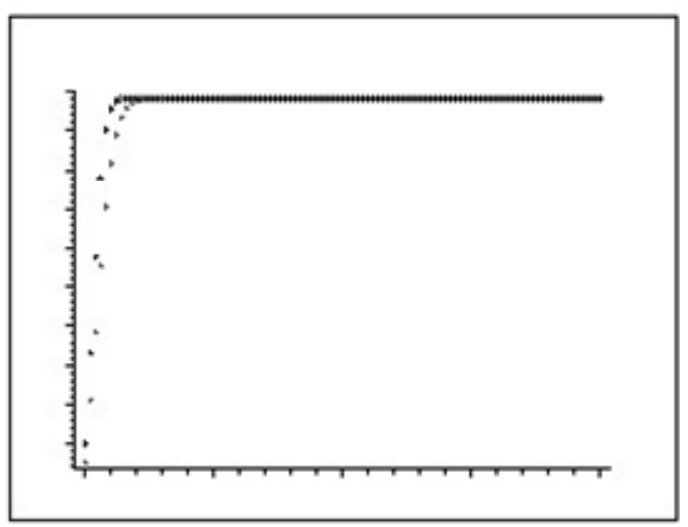

(b)

Figure 2. (a) $x_{1}(k)$ versus $k$ before being stabilized; (b) $x_{1}(k)$ versus $k$ after being stabilized for $q=0.5$ and $q=0.3$.

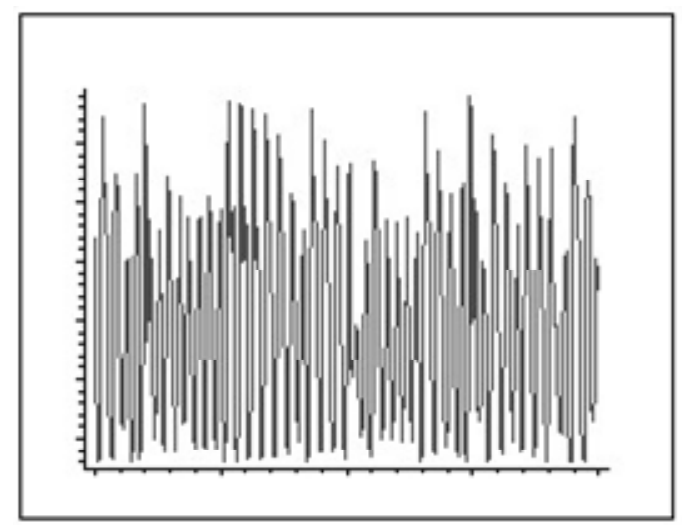

(a)

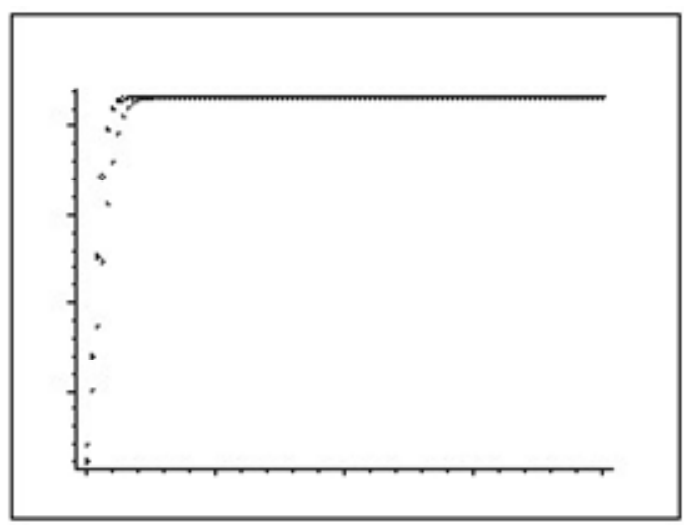

(b)

Figure 3. (a) $x_{2}(k)$ versus $k$ before being stabilized; (b) $x_{2}(k)$ versus $k$ after being stabilized for $q=0.5$ and $q=0.3$.

$$
\left\{\begin{array}{l}
y_{1}(k+1)=a_{3} \delta y_{2}(k)+\left(a_{4} \delta+1\right) y_{1}(k), \\
y_{2}(k+1)=a_{2} \delta y_{3}(k)+a_{1} \delta y_{1}(k)+y_{2}(k), \\
y_{3}(k+1)=a_{5} \delta+a_{6} \delta y_{2}(k) y_{3}(k)+\left(a_{7} \delta+1\right) y_{3}(k),
\end{array}\right.
$$

which was derived from the generalized Rössler system via a first-order difference algorithm [15]

We take the fixed point $(0.09610764055$, $0.4420951466,0.9130225853)$ as our research object, that is $\left(y_{1 f}, y_{2 f}, y_{3 f}\right)=(0.09610764055,0.4420951466$, 0.9130225853 ).

Following the procedure above, the Jacobian matrix of 
map (13) is

$$
J=\left(\begin{array}{ccc}
a_{4} \delta+1 & a_{3} \delta & 0 \\
a_{1} \delta & 1 & a_{2} \delta \\
0 & a_{6} \delta y_{3 f} & a_{6} \delta y_{2 f}+a_{7} \delta+1
\end{array}\right) .
$$

Here we let $a_{1}=-1.9, a_{2}=0.2, a_{3}=0.5, a_{4}=-2.3, a_{5}$ $=2, a_{6}=-0.6, a_{7}=-1.9$ and $\delta=1$.

From (6), choosing $q=0.5$ and $q=-0.2$ respectively, the matrix $M$ at the fixed point $(0.09610764055$, $0.4420951466,0.9130225853)$ is correspondingly obtained as following

$$
M=\left(\begin{array}{ccc}
-0.9762747382 & 0.2344378413 & 0.02165450397 \\
-0.8908637970 & 0.0784140703 & 0.09961071835 \\
0.2253899842 & -0.2728405070 & -0.7942822117
\end{array}\right),
$$

and

$$
M=\left(\begin{array}{ccc}
-0.9430593722 & 0.5626508191 & 0.05197080956 \\
-2.138073113 & 1.588193768 & 0.2390657240 \\
0.5409359615 & -0.6548172166 & -0.562773086
\end{array}\right)
$$

From (2), respectively substitute (15) and (16) into (13), one can obtain

$$
\left\{\begin{aligned}
y_{1}(k+1)= & 0.0237252618\left[a_{3} \delta y_{2}(k)+a_{4} \delta y_{1}(k)+y_{1}(k)\right]+0.9762747382 y_{1}(k)+0.2344378412 \\
& \times\left[a_{2} \delta y_{3}(k)+a_{1} \delta y_{1}(k)\right]+0.02165450397\left[a_{5} \delta+a_{6} \delta y_{2}(k) y_{3}(k)+a_{7} \delta y_{3}(k)\right], \\
y_{2}(k+1)= & 1.07841407\left[a_{2} \delta y_{3}(k)+a_{1} \delta y_{1}(k)\right]+y_{2}(k)-0.890863797\left[a_{3} \delta y_{2}(k)+a_{4} \delta y_{1}(k)\right] \\
& +0.09961071835\left[a_{5} \delta+a_{6} \delta y_{2}(k) y_{3}(k)+a_{7} \delta y_{3}(k)\right], \\
y_{3}(k+1)= & 0.2057177883\left[a_{5} \delta+a_{6} \delta y_{2}(k) y_{3}(k)+\left(a_{7} \delta+1\right) y_{3}(k)\right]+0.2253899842\left[a_{3} \delta y_{2}(k)\right. \\
& \left.+a_{4} \delta y_{1}(k)\right]-0.272840507\left[a_{2} \delta y_{3}(k)+a_{1} \delta y_{1}(k)\right]+0.7942822117 y_{3}(k),
\end{aligned}\right.
$$

and

$$
\left\{\begin{aligned}
y_{1}(k+1)= & 0.0569406278\left[a_{3} \delta y_{2}(k)+a_{4} \delta y_{1}(k)+y_{1}(k)\right]+0.9430593722 y_{1}(k)+0.5626508191 \\
& \times\left[a_{2} \delta y_{3}(k)+a_{1} \delta y_{1}(k)\right]+0.05197080956\left[a_{5} \delta+a_{6} \delta y_{2}(k) y_{3}(k)+a_{7} \delta y_{3}(k)\right] \\
y_{2}(k+1)= & 2.588193768\left[a_{2} \delta y_{3}(k)+a_{1} \delta y_{1}(k)\right]+y_{2}(k)-2.138073113\left[a_{3} \delta y_{2}(k)+a_{4} \delta y_{1}(k)\right] \\
& +0.239065724\left[a_{5} \delta+a_{6} \delta y_{2}(k) y_{3}(k)+a_{7} \delta y_{3}(k)\right] \\
y_{3}(k+1)= & 0.4937226914\left[a_{5} \delta+a_{6} \delta y_{2}(k) y_{3}(k)+\left(a_{7} \delta+1\right) y_{3}(k)\right]+0.5409359615\left[a_{3} \delta y_{2}(k)\right. \\
& \left.+a_{4} \delta y_{1}(k)\right]-0.6548172166\left[a_{2} \delta y_{3}(k)+a_{1} \delta y_{1}(k)\right]+0.5062773086 y_{3}(k)
\end{aligned}\right.
$$

The numerical results are shown in the followed figures. The orbit of 3-dimension discrete time hyperchaotic system is given by Figure 4(a). In Figure 4(b), three orbits starting from different initial points are stabilized to the fixed point $(0.09610764055,0.4420951466$, $0.9130225853)$.

We can also get the result that 3-dimension discrete time hyperchaotic system is stabilized. In Figures 5-7, the stabilized orbits of $y_{1}(k), y_{2}(k), y_{3}(k)$ versus $t_{k}$ are plotted contrasting with the ones before being stabilized, respectively.

\section{Conclusion}

In summary, we have introduced a method to stabilize unstable discrete systems, which does not require any adjustable control parameters of the system. 2-dimension discrete Fold system and 3-dimension discrete hyperchaotic system are stabilized to fixed points respectively. From the process we finish, it is shown that stabilizing the unstable discrete systems neither requires a prior analytical knowledge of the underlying system nor any adjustable control parameters in advance. Numerical simulations are then provided to show the effectiveness 


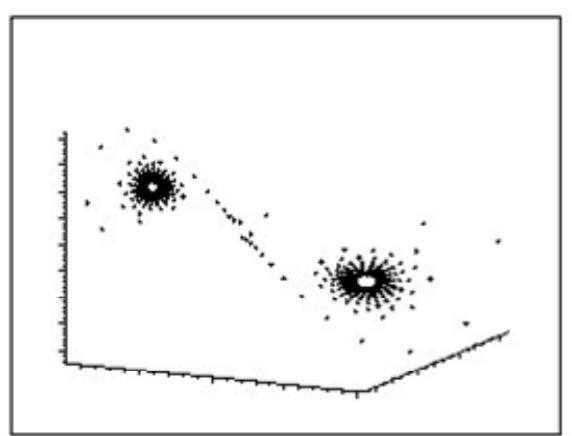

(a)

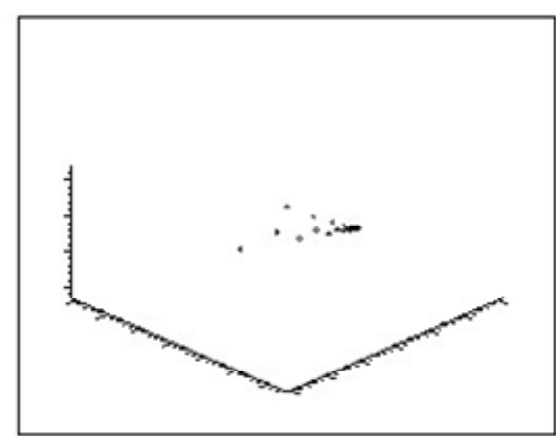

(b)

Figure 4. (a) 3-dimension discrete time hyperchaotic system; (b) Three orbits starting from different initial points are stabilized to the fixed point $(0.09610764055,0.4420951466,0.9130225853)$, for $q=0.5$.

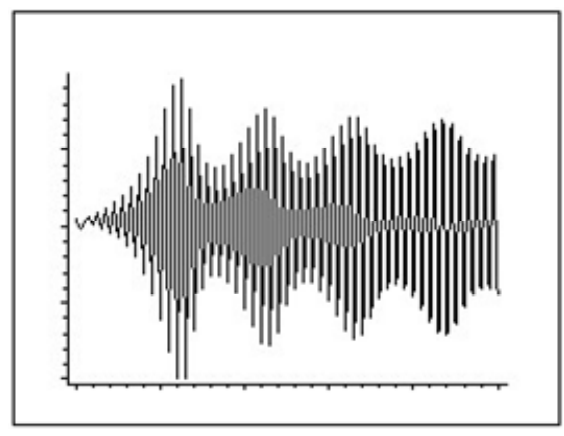

(a)

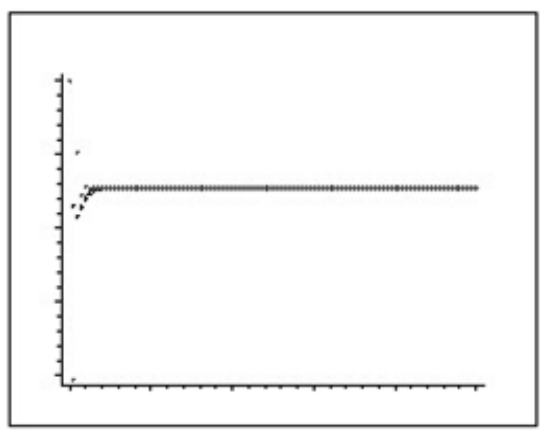

(b)

Figure 5. (a) $y_{1}(k)$ versus $k$ before being stabilized; (b) $y_{1}(k)$ versus $k$ after being stabilized.

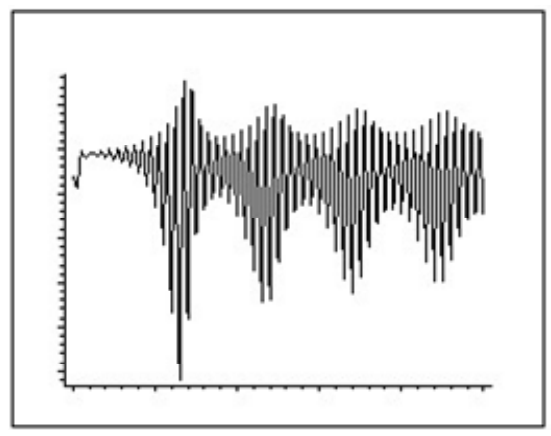

(a)

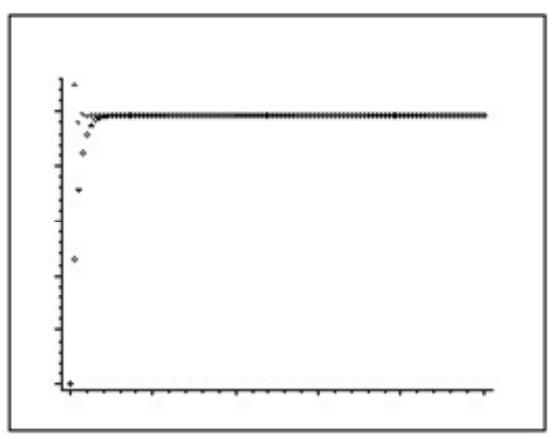

(b)

Figure 6. (a) $y_{2}(k)$ versus $k$ before being stabilized; (b) $y_{2}(k)$ versus $k$ after being stabilized.

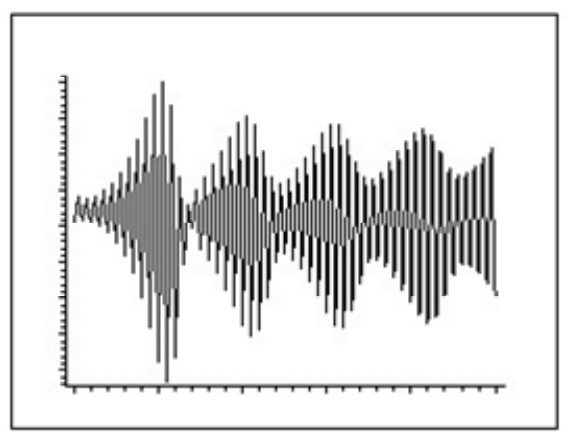

(a)

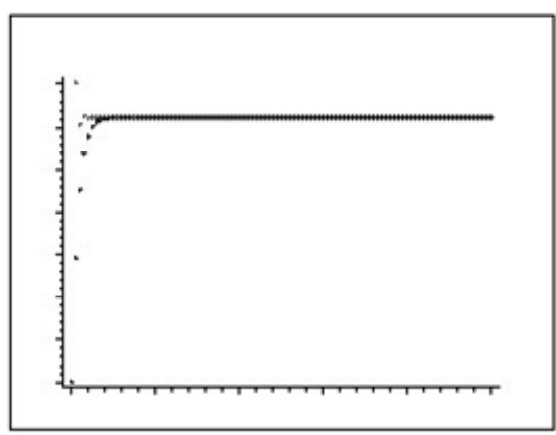

(b)

Figure 7. (a) $y_{3}(k)$ versus $k$ before being stabilized; (b) $y_{3}(k)$ versus $k$ after being stabilized. 
and feasibility of the proposed chaos and hyperchaos controlling Scheme.

\section{Acknowledgements}

The authors are grateful to the reviewers for their valuable comments and suggestions.

\section{REFERENCES}

[1] E. Ott, C. Grebogi and J. A. York, "Controlling Chaos," Physical Review Letters, Vol. 64, No. 11, 1990, pp. 11961199.

[2] D. Liu, L. Liu and Y. Yang, " $H_{\infty}$ Control of DiscreteTime Singularly Perturbed Systems via Static Output Feedback," Abstract and Applied Analysis, Vol. 2013, 2013, Article ID: 528695.

[3] Z. Wang, H. T. Zhao and X. Y. Kong, "Delayed Feedback Control and Bifurcation Analysis of an Autonomy System," Abstract and Applied Analysis, Vol. 2013, 2013, Article ID: 167065.

[4] G. R. Chen and J. H. LU, "Dynamic Analysis, Control and Synchronization of Lorenz System," Science Publishing Company, Beijing, 2003.

[5] B. Peng, V. Petro and K. Showalter, "Controlling Chemical Chaos," The Journal of Physical Chemistry, Vol. 95, No. 13, 1991, pp. 4957-4961. http://dx.doi.org/10.1021/j100166a013

[6] H. Salarieh and A. Alasty, "Control of Stochastic Chaos Using Sliding Mode Method," Journal of Computational and Applied Mathematics, Vol. 225, No. 1, 2009, pp. 135-145. http://dx.doi.org/10.1016/j.cam.2008.07.032

[7] K. Pyragas, "Continuous Control of Chaos by Self-Controlling Systems," Physical Letter A, Vol. 170, No. 6,
1992, pp. 421-428. http://dx.doi.org/10.1016/0375-9601(92)90745-8

[8] E. H. Abed, H. O. Wang and R. C. Chen, "Stabilization of Period Doubling Bifurcations and Implications for Control of Chaos," Physica D, Vol. 70, No. 1-2, 1994, pp. 154-164.

http://dx.doi.org/10.1016/0167-2789(94)90062-0

[9] K. A. Mirus and J. C. Sprott, "Controlling Chaos in Lowand High-Dimensional Systems with Periodic Parametric Perturbations," Physical Review E, Vol. 59, No. 5, 1999 , pp. 5313-5324.

http://dx.doi.org/10.1103/PhysRevE.59.5313

[10] X. Li, Y. Chen and Z. B. Li, "Function Projective Synchronization of Discrete-Time Chaotic Systems," Zeitschrift für Naturforschung Section A-A Journal of Physical Scienses, Vol. 63, No. 1-2, 2008, pp. 7-14.

[11] L. Yang, Z. R. Liu and J. M. Mao, "Controlling Hyperchaos," Physical Review Letters, Vol. 84, No. 1, 2000, pp. 67-70. http://dx.doi.org/10.1103/PhysRevLett.84.67

[12] S. L. Bu, S. Q. Wang and H. Q. Ye, "Stabilizing Unstable Discrete Systems," Physical Review E, Vol. 64, 2001, Article ID: 046209.

[13] X. Li and Y. Chen, "Stabilizing of Two-Dimensional Discrete Lorenz Chaotic System and Three-Dimensional Discrete Rössler Hyperchaotic System," Chinese Physics Letters, Vol. 26, No. 9, 2009, Article ID: 090503.

[14] M. Itoh, T. Yang and L. O. Chua, "Conditions for Impulsive Synchronization of Chaotic and Hyperchaotic Systems," International Journal of Bifurcation and Chaos, Vol. 11, No. 2, 2001, pp. 551-560. http://dx.doi.org/10.1142/S0218127401002262

[15] X. Y. Wang, "Chaos in Complex Nonlinear Systems," Publishing House of Electronics Industry, Beijing, 2003. 